\title{
ASPECTOS AGRONÔMICOS DE LEGUMINOSAS PARA ADUBAÇÃO VERDE NO CERRADO DO ALTO VALE DO JEQUITINHONHA ${ }^{(1)}$
}

\author{
Ricardo Borges Teodoro ${ }^{(2)}$, Fábio Luiz de Oliveira ${ }^{(3)}$, Diego Mathias Natal da \\ Silva $^{(4)}$, Claudenir Fávero ${ }^{(3)}$ \& Mateus Augusto Lima Quaresma $^{(5)}$
}

\begin{abstract}
RESUMO
O uso intensivo e inadequado dos solos acelera sua degradação, sendo necessária a intervenção por meio de práticas conservacionistas para restaurar a capacidade produtiva dos mesmos. O objetivo deste estudo foi avaliar o comportamento e desenvolvimento de diferentes leguminosas utilizadas como adubos verdes em solos de Cerrado, Alto Vale do Jequitinhonha, em Turmalina, MG. O delineamento experimental adotado foi em blocos ao acaso, com sete tratamentos e quatro repetições, sendo os tratamentos constituídos pelas leguminosas: mucuna-cinza (Mucuna nivea), mucuna-preta (Mucuna aterrima), lablabe (Dolichos lablab), feijão-de-porco (Canavalia ensiformis), Crotalaria juncea, Crotalaria spectabilis e guandu-anão (Cajanus cajan). O ciclo precoce de C.juncea, $C$. spectabilis e feijão-de-porco favorece a inserção destes nos sistemas de cultivo. Aos 40 dias, o feijão-de-porco e mucuna-cinza já cobriam o solo, com 67 e $63 \%$; já o guandu-anão e $C$. juncea apresentaram os maiores desenvolvimentos, nesse período. Os teores de $\mathrm{N}, \mathrm{P}$ e $\mathrm{K}$ tendem a diminuir nas avaliações realizadas nas diferentes fases vegetativas, o que contribui para melhor escolha da época de manejo das leguminosas. Crotalaria juncea, mucuna-cinza, feijão-de-porco e mucuna-preta foram as leguminosas que se destacaram na produção de matéria seca, o que torna essas espécies promissoras para adubação verde na região. As leguminosas, em sua maioria, apresentam potencial para reciclagem dos macronutrientes e aporte de $\mathrm{N}$ aos sistemas de produção.
\end{abstract}

Termos de indexação: adubos verdes, ciclagem de nutrientes, manejo do solo, plantas de cobertura.

\footnotetext{
(1) Parte da Dissertação de Mestrado do primeiro autor apresentada a Universidade Federal dos Vales do Jequitinhonha e Mucuri - UFVJM. Projeto financiado pelo CNPq e FAPEMIG. Recebido para publicação em maio de 2010 e aprovado em janeiro de 2011.

(2) Mestre em Produção Vegetal, Departamento de Agronomia. Universidade Federal dos Vales do Jequitinhonha e Mucuri UFVJM. Campus I, Rua da Glória 187, Centro, CEP 39100-000 Diamantina (MG). Bolsista CNPq. E-mail: ricardo.agronomia@hotmail.com

(3) Professor Adjunto, Departamento de Agronomia. UFVJM. E-mails: fabiocapi@yahoo.com.br; prufvjm@yahoo.com.br

(4) Mestrando em Produção Vegetal, Departamento de Agronomia. UFVJM. Bolsista CAPES. E-mail:diegoufvjm@yahoo.com

(5) Graduando em Agronomia, Departamento de Agronomia. UFVJM. E-mail: mateusveio@yahoo.com.br
} 


\title{
SUMMARY: AGRONOMIC ASPECTS OF LEGUMINOUS TO GREEN FERTILIZATION IN THE CERRADO OF THE HIGH JEQUITINHONHA VALLEY
}

\begin{abstract}
The intensive and inadequate use of soils accelerates its degradation, requiring the intervention by means of conservation practices to restore the productive capacity of the same. The aim of this study was to evaluate the behavior and development of different legumes used as green manure in soils of the Cerrado, High Jequitinhonha Valley in Turmalina, MG. The experimental design was in randomized blocks with seven treatments and four replications, the treatments were the leguminous: Mucuna nivea, Mucuna aterrima, Dolichos lablab, Canavalia ensiformis, Crotalaria juncea, Crotalaria spectabilis and Cajanus cajan. The early cycle of Crotalaria juncea, Crotalaria spectabilis and Canavalia ensiformis, favors the insertion of these in cropping systems. At 40 days, the Canavalia ensiformis and Mucuna nivea stood out to cover the soil with 67 and $63 \%$, the Cajanus cajan and Crotalaria juncea presented the highest development in this period. The contents of $N, P$ and $K$ tend to decrease in the evaluations realized in different vegetative stages, which contributes to better choice of the time management of legumes. Crotalaria juncea, Mucuna nivea, Canavalia ensiformis and Mucuna aterrima, were the leguminous that stood out for fitomass production, making these promising species for green manure in the region. The leguminous in your mostly, presents potential for recycling of macronutrients and the contribution of $N$ to production systems.
\end{abstract}

Index terms: green manuring, nutrient cycling, soil management, cover crops.

\section{INTRODUÇÃO}

A ocupação do Cerrado tem ocorrido de forma rápida, com base em sistemas de produção intensivos, que têm aumentado os processos de degradação dos solos (Silva et al., 1994). Esses processos resultam em impactos que muitas vezes geram a ineficiência dos sistemas agrícolas, sendo frequente a necessidade de intervenções por meio de práticas conservacionistas para manutenção do potencial produtivo do solo. Nesse sentido, a adubação verde torna-se fundamental para a região de Cerrado, pois ela promove proteção, melhoria e manutenção da qualidade do solo, além de aumentos consideráveis dos teores de matéria orgânica e nutrientes, beneficiando os agroecossistemas (Carvalho et al., 1999; Leite et al., 2010). Entre as características desejáveis para a seleção de espécies para adubação verde, destacam-se a produção de matéria seca, capacidade de incrementar nutrientes pela simbiose com microrganismos, cobertura do solo e reciclagem de macronutrientes (Chaves \& Calegari, 2001).

Entre as várias espécies de famílias botânicas que podem ser cultivadas como adubos verdes, destacamse aquelas da família Fabaceae. As leguminosas, além de proporcionarem benefícios semelhantes aos de outras espécies, apresentam a capacidade de acumular $\mathrm{N}$ pela fixação biológica (Silva et al., 2009). No entanto, para viabilizar o uso de plantas condicionadoras de solo em áreas de Cerrado, é necessário associar propriedades agronômicas com as condições edafoclimáticas, além dos sistemas de manejo (incorporação e plantio direto) e de cultivo (rotação, sucessão e consórcio), visando melhorar o aproveitamento do potencial das espécies vegetais utilizadas (Carvalho \& Amabile, 2006).
Uma das principais propriedades morfoagronômicas utilizados para avaliar os adubos verdes é a produção de matéria seca. No bioma Cerrado, os rendimentos de matéria seca variam com o genótipo, a época de semeadura, as condições edafoclimáticas e as práticas de manejo, bem como com a população de plantas em uso (Amabile et al., 1996, 2000; Carvalho et al., 1999). Pesquisas em solos de região de Cerrado sugerem a necessidade de atingir, em programas de manejo e cultivo, a produção de 6 a $12 \mathrm{Mg} \mathrm{ha}^{-1}$, pelo fato de a decomposição do material depositado na superfície ser mais elevada nas regiões de clima tipicamente tropical (Darolt, 1998; Alvarenga et al., 2001).

Atualmente, entre as diversas leguminosas utilizadas para adubação verde na região dos Cerrados, destacam-se: a Crotalaria juncea, Crotalaria spectabilis, mucunas cinza e preta (Mucuna nívea e Mucuna aterrima, respectivamente), lablabe (Dolichos lablab), feijão-de-porco (Canavalia ensiformis) e guandu-anão (Cajanus cajan) (Arf et al., 2000; Santos \& Campelo Junior, 2003; Cazetta et al., 2005; Silveira et al., 2005; Torres et al., 2005; Suzuki $\&$ Alves, 2006; Carneiro et al., 2008).

A Crotalaria juncea responde ao fotoperíodo; o atraso da semeadura reduz os rendimentos de matéria seca (Amabile et al., 2000). A Crotalaria spectabilis destaca-se na região de Cerrado por seu ciclo relativamente curto, que possibilita semeadura anterior à da cultura principal; contudo, em áreas de Cerrado, somente se adapta ao cultivo no período chuvoso (Carvalho \& Amabile, 2006).

A mucuna-preta apresenta desenvolvimento vegetativo eficiente e acentuada rusticidade para o Cerrado, adaptando-se bem às condições de deficiência 
hídrica e de temperaturas altas (Amabile et al., 2000). A mucuna-cinza destaca-se pela capacidade de cobertura do solo em áreas de Cerrado (Carvalho, 2005), permanecendo sobre ele por longo período; essa característica, associada à elevada produção de matéria seca e à alta velocidade de crescimento, confere-lhe potencial de uso para cobertura de solo (Sodré Filho et al., 2004).

O lablabe, no Cerrado, adapta-se ao cultivo no período de chuva, pois é sensível ao fotoperíodo e ao déficit hídrico. Apresenta ciclo longo: aproximadamente 156 dias até a floração (Carvalho et al.,1999). Calegari et al. (1993) relatam que, em solo com fertilidade baixa e $\mathrm{pH}$ ácido, normalmente seu crescimento é lento e a produção de matéria seca menor.

O feijão-de-porco é uma planta adaptada a condições ambientais bem adversas, suportando desde o clima árido e seco das regiões semiáridas até o de regiões com florestas tropicais. No Cerrado do Brasil Central, pode ser semeado até no final do período de chuvas, devido a sua resistência à seca e por não apresentar sensibilidade ao fotoperíodo (Calegari et al., 1993; Carvalho et al.,1999). Em sistemas agrícolas nas áreas de Cerrado, é utilizado em consórcio com milho e culturas perenes, já que tolera o sombreamento parcial (Carvalho \& Amabile, 2006).

O guandu-anão, de ciclo anual ou semiperene, é pouco exigente em relação à fertilidade do solo e adapta-se a ampla faixa de precipitação pluvial; é resistente à seca, mostrando-se bem adaptado aos solos que predominam no Bioma Cerrado (Tanaka, 1981). Promove a liberação do $\mathrm{P}$ comumente adsorvido aos óxidos e hidróxidos de Fe nos solos do Cerrado (Ae et al., 1990). As épocas de semeadura podem alterar a absorção de $\mathrm{N}$ e $\mathrm{P}$ nos solos do Cerrado (Amabile et al., 2000).

Apesar da importância, a adubação verde é uma prática pouco empregada nos Cerrados do Alto Vale do Jequitinhonha; assim, para utilizá-la, é necessário o conhecimento de informações básicas, como as espécies mais adequadas e os respectivos parâmetros agronômicos.

Este trabalho teve como objetivo avaliar o comportamento e desenvolvimento de diferentes leguminosas utilizadas como adubos verdes (Mucuna nivea, Mucuna aterrima, Dolichos lablab, Canavalia ensiformis, Crotalaria juncea, Crotalaria spectabilis e Cajanus cajan) nas condições de solo de Cerrado, no Alto Vale do Jequitinhonha, em Turmalina (MG).

\section{MATERIAL E MÉTODOS}

O presente trabalho foi realizado no Centro de Educação e Arte de Turmalina-CEART, no município de Turmalina-MG, na região de Cerrado, no Alto Vale do Jequitinhonha $\left(17^{\circ} 23^{\prime} 37\right.$ " S e $42^{\circ} 35^{\prime} 22$ ” W, com $855 \mathrm{~m}$ de altitude). As médias de precipitação pluvial, temperatura mínima e máxima foram de $1.418 \mathrm{~mm}, 21,7$ e $33,2^{\circ} \mathrm{C}$, respectivamente, no período de $21 / 11 / 2008$ a $12 / 5 / 2009$.

O solo da área utilizada no experimento foi classificado como Latossolo Vermelho-Amarelo distrófico (Embrapa, 2006). Foram retiradas da área amostras de solo $(0-20 \mathrm{~cm})$, com as seguintes características químicas e granulométricas: $\mathrm{pH}$ em água de 4,3;13,8 $\mathrm{mg} \mathrm{dm}^{-3}$ de $\mathrm{P}_{\text {Mehlich } 1} ; 180 \mathrm{mg} \mathrm{dm}^{-3}$ de $\mathrm{K} ; 1,0 \mathrm{cmol}_{\mathrm{c}} \mathrm{dm}^{-3}$ de $\mathrm{Ca} ; 0,4 \mathrm{cmol}_{\mathrm{c}} \mathrm{dm}^{-3} \mathrm{de} \mathrm{Mg}$; $1,4 \mathrm{cmol}_{\mathrm{c}} \mathrm{dm}^{-3} \mathrm{de} \mathrm{Al}$; saturação por bases igual a $8 \%$; areia, $34 \%$; silte, $10 \%$; e argila, $56 \%$.

O delineamento experimental foi em blocos ao acaso, com sete tratamentos e quatro repetições, sendo os tratamentos constituídos pelas leguminosas: mucuna-cinza (Mucuna nivea), mucuna-preta (Mucuna aterrima), lablabe (Dolichos lablab), feijãode-porco (Canavalia ensiformis), Crotalaria juncea, Crotalaria spectabilis e guandu-anão (Cajanus cajan).

A área em estudo encontrava-se em pousio, coberta por plantas espontâneas por período superior a dois anos, tendo sido anteriormente cultivada com hortaliças. Realizou-se aplicação a lanço e sem incorporação de $2,5 \mathrm{Mg} \mathrm{ha}^{-1}$ de calcário dolomítico (PRNT de 80 \%) na área, 30 dias antes da semeadura. Antes da semeadura foi realizada capina manual em toda a área do experimento, sendo as leguminosas semeadas em 21 de novembro de 2008. O espaçamento entre sulcos e a densidade de semeadura por metro linear utilizados para as leguminosas foram de $40 \mathrm{~cm}$, com 10 sementes para mucuna-cinza, mucuna-preta e feijão-de-porco e 20 sementes para o guandu-anão, Crotalaria juncea, Crotalaria spectabilis e lablabe; a profundidade de plantio foi de 2 a $5 \mathrm{~cm}$, com semeadura manual. A área de cada parcela foi de $4 \mathrm{~m}^{2}(2 \times 2 \mathrm{~m})$, sendo considerada área útil os $2 \mathrm{~m}^{2}$ centrais.

As características avaliadas nas leguminosas foram: duração do ciclo até o florescimento; taxa de cobertura do solo; potencial de deposição de folhas e aporte de macronutrientes, por meio da senescência de folhas; produção total de matéria seca; e acúmulo de macronutrientes na parte aérea.

O ciclo das espécies foi determinado quando as parcelas apresentavam mais de $50 \%$ das plantas em pleno florescimento. A taxa de cobertura do solo foi determinada aos 30, 60 e 90 dias após a semeadura (DAS) das leguminosas, pelo método do número de interseções descrito por Fávero et al. (2001), utilizandose um quadro de madeira de $1 \mathrm{~m}^{2}$ contendo uma rede de barbantes, espaçados de $10 \mathrm{~cm}$, definindo 100 pontos, sobre a área em que se queria determinar a cobertura. A interseção entre dois barbantes perpendiculares define um ponto e representa uma área, conforme espaçamento adotado. Conta-se então o número de interseções que estão sobre a vegetação. O somatório desses pontos, que significa o somatório das áreas que esses pontos representam, em relação à área total dos pontos do conjunto, fornece a percentagem de cobertura do solo. Esse método foi 
aplicado apenas para mucuna-preta, mucuna-cinza, lablabe e feijão-de-porco; para as espécies de crescimento arbustivo ereto, Crotalaria juncea, Crotalaria spectabilis e guandu-anão, não se determinou a taxa de cobertura do solo, devido ao difícil manuseio e aos possíveis danos a serem provocados às plantas com essa característica. Entretanto, avaliou-se o desenvolvimento destas nas referidas datas, determinando-se a alturas delas. Para avaliação da altura, foi usada uma fita milimetrada, medindo-se a altura do dossel de 10 plantas, a partir da superfície do solo até o ápice.

O potencial de deposição de folhas e aporte de macronutrientes pelas leguminosas foi observado quantificando-se a matéria seca senescente depositada sobre o solo e estimando-se o acúmulo de macronutrientes nessa matéria seca, na área útil de $1 \mathrm{~m}^{2}$. As coletas foram realizadas aos 60,90 e 120 DAS.

Para determinação do acúmulo de matéria seca na parte aérea, foi realizado o corte das plantas no momento em que as parcelas apresentavam $50 \%$ ou mais de plantas em florescimento, quando se cortou metade da área útil em cada parcela, sendo as plantas cortadas rente à superfície do solo, fazendo-se, em seguida, a pesagem do material vegetal. $\mathrm{O}$ acúmulo da matéria seca foi determinado indiretamente, por meio da umidade do material verde, aferida em amostras de $100 \mathrm{~g}$, que foram secas em estufa com ventilação de ar forçada a $65^{\circ} \mathrm{C}$, por $72 \mathrm{~h}$, até atingir massa constante. Nessa matéria seca também foram estimados os teores e acúmulos de macronutrientes. Posteriormente, quando as plantas se encontravam na fase de frutificação, foi efetuado o corte da outra metade da parcela. As amostras coletadas foram secas em estufa com ventilação forçada de ar a $65{ }^{\circ} \mathrm{C}$, por $72 \mathrm{~h}$, até atingirem massa constante, quantificandose, em seguida, os teores dos macronutrientes na matéria seca de cada espécie.
Nas análises químicas dos materiais vegetais, o teor de $\mathrm{N}$ foi determinado após digestão sulfúrica e destilação em Kjeldahl (Bremner \& Mulvaney, 1982). Os teores de P, K, Ca e Mg foram determinados após digestão nítrico-perclórica (Bataglia et al., 1983), sendo o P determinado em espectrofotômetro a partir de formação da cor azul do complexo fosfato-molibdato em meio sulfúrico, na presença de ácido ascórbico como redutor; o K, por fotômetro de chama; e o $\mathrm{Ca}$ e $\mathrm{Mg}$, em espectrofotômetro de absorção atômica (Embrapa, 1997).

As análises estatísticas foram realizadas com auxílio do programa estatístico SISVAR (Ferreira, 2008). Os dados foram submetidos à análise de variância pelo teste $\mathrm{F}$, e as médias, comparadas pelo teste de Scott-Knott a $5 \%$.

\section{RESULTADOS E DISCUSSÃO}

Houve diferença significativa entre as espécies no período de duração do ciclo. Crotalaria juncea, Crotalaria spectabilis e feijão-de-porco apresentaram menores ciclos em relação às demais (Quadro 1). Esses resultados favorecem a inserção deles em sistemas de cultivos com rotação e sucessão de culturas. O lablabe, a mucuna-cinza e a mucuna-preta apresentaram ciclos mais longos, o que pode inviabilizar a introdução deles em sistemas de cultivo com rotação e sucessão de culturas, pois isso implicaria permanência por período maior na área de cultivo, o que muitas vezes não é interessante para o agricultor, em decorrência da menor otimização da área. Entretanto, apresentam potencial para utilização em consórcio com culturas de ciclo semelhante. Calegari (1995) ressalta a importância dos ciclos mais curtos para acúmulo precoce de matéria seca em espécies usadas na

Quadro 1. Duração do ciclo vegetativo até o pleno florescimento das leguminosas, taxa de cobertura do solo das leguminosas de crescimento volúvel, altura e taxa de desenvolvimento das leguminosas arbustivas, em diferentes períodos

\begin{tabular}{|c|c|c|c|c|c|c|c|c|c|c|c|}
\hline \multirow{3}{*}{ Espécie } & \multirow{3}{*}{$\begin{array}{l}\text { Ciclo } \\
\text { (dia) }\end{array}$} & \multicolumn{4}{|c|}{ Cobertura do solo (dia) } & \multicolumn{6}{|c|}{ Altura e } \\
\hline & & \multirow[t]{2}{*}{40} & \multirow[t]{2}{*}{60} & \multirow[t]{2}{*}{90} & \multirow[t]{2}{*}{120} & \multicolumn{2}{|c|}{40 dias } & \multicolumn{2}{|c|}{60 dias } & \multicolumn{2}{|c|}{ Flor } \\
\hline & & & & & & Altura & $\overline{\text { Taxa }}$ & Altura & $\overline{\text { Taxa }}$ & Altura & $\overline{\text { Taxa }}$ \\
\hline $\mathrm{Ll}$ & $163 \mathrm{a}^{(1)}$ & 46 & 97 & 100 & 100 & * & * & * & * & * & * \\
\hline $\mathrm{Mp}$ & $156 \mathrm{a}$ & 42 & 95 & 100 & 100 & * & * & * & * & * & * \\
\hline $\mathrm{Mc}$ & $147 \mathrm{a}$ & 63 & 100 & 100 & 100 & * & * & * & * & * & * \\
\hline $\mathrm{Fp}$ & $97 \mathrm{c}$ & 67 & 100 & 100 & $*$ & * & * & * & * & * & * \\
\hline $\mathrm{Cj}$ & $88 \mathrm{c}$ & $*$ & $*$ & $*$ & * & $123 \mathrm{a}$ & 47,7 & $173 \mathrm{a}$ & 19,4 & $258 \mathrm{a}$ & 32,9 \\
\hline $\mathrm{Ce}$ & $92 \mathrm{c}$ & * & * & * & * & $39 \mathrm{c}$ & 31,5 & $77 \mathrm{~b}$ & 30,6 & $124 \mathrm{~b}$ & 37,9 \\
\hline $\mathrm{Ga}$ & $119 \mathrm{~b}$ & $*$ & $*$ & * & $*$ & $55 \mathrm{c}$ & 51,4 & $96 \mathrm{~b}$ & 38,3 & $107 \mathrm{~b}$ & 10,3 \\
\hline $\mathrm{CV}(\%)$ & 6,10 & - & - & - & - & 6,08 & - & 18,30 & - & 9,42 & - \\
\hline
\end{tabular}

(1) Valores seguidos de letras iguais, dentro da coluna, não diferem entre si teste de Scott-Knott $(p<0,05)$. Ll: lablabe; Mp: mucunapreta; Mc: mucuna-cinza; Fp: feijão-de-porco; Cj: Crotalaria juncea; Ce: Crotalaria spectabilis; Ga: guandu-anão; * Não avaliado. 
adubação verde, principalmente em sistemas com frequente rotação de culturas.

Ao avaliar a taxa de cobertura do solo pelas leguminosas herbáceas volúveis, observou-se que feijão-de-porco e mucuna-cinza apresentaram cobertura em torno de 67 e $63 \%$, respectivamente, aos 40 DAS, cobrindo totalmente o solo aos 60 dias, ao passo que o lablabe e a mucuna-preta apresentaram em torno de $40 \%$ aos 40 dias e cobertura plena do solo após 60 dias, o que demonstra a menor velocidade dessas duas últimas em cobrir o solo (Quadro 1). Comportamento semelhante foi observado por Fávero et al. (2001) e Perin et al. (2000), que destacam a velocidade de cobertura do solo para o feijão-de-porco e a mucuna-cinza nos primeiros 30 DAS. Os resultados do presente trabalho demonstram a alta eficiência das espécies para cobertura do solo nos primeiros 40 DAS, o que tem implicações práticas do uso dessas espécies em solos degradados e, ou, pousio na região, podendo reduzir assim o impacto das gotas da chuva na superfície do solo.

O guandu-anão concentra seu crescimento e desenvolvimento nos primeiros $60 \mathrm{DAS}$, com altura média das plantas de $96 \mathrm{~cm}$. Esse comportamento demonstra que a espécie direciona grande parte da sua energia produzida para o seu crescimento nos primeiros 60 DAS, sendo seu crescimento reduzido a partir desse período (Quadro 1). Para Crotalaria juncea, a taxa de crescimento é mais acelerada até os 40 DAS, reduzindo entre os 40 e 60, e após os 60 DAS volta a acelerar novamente (Quadro 1). Oliveira \& Gosch (2007) estudaram o comportamento de leguminosas arbustivas em região com características edafoclimáticas semelhantes e também observaram pico de crescimento da Crotalaria juncea após os 60 dias. Wallis et al. (1981) atribuem esse desenvolvimento fenológico à interação fotoperíodo $\mathrm{x}$ temperatura e época de semeadura e latitude, corroborando a pesquisa de Amabile et al. (2000), que mencionam novembro como época favorável ao plantio dessa espécie na região de Cerrado. Crotalaria spectabilis comporta-se de forma diferente, sendo a sua taxa de crescimento praticamente constante nos diferentes períodos avaliados (Quadro 1). O comportamento do guandu-anão e da Crotalaria juncea, de concentrar o seu crescimento nos primeiros 40 DAS, reflete-se no seu rápido estabelecimento e crescimento, fato importante na ocupação de espaços, diminuindo a incidência de plantas espontâneas, no aumento da proteção do solo, no acúmulo de matéria seca e no aporte de nutrientes.

O potencial de deposição de matéria seca senescente pelas leguminosas (Quadro 2) variou de acordo com o crescimento das espécies. Aos 60 DAS, as espécies

Quadro 2. Deposição de matéria seca e ciclagem macronutrientes por ocasião de diferentes coletas de material senescente das leguminosas até os 120 dias

\begin{tabular}{|c|c|c|c|c|c|c|}
\hline Espécie & Matéria seca & $\mathbf{N}$ & $\mathbf{P}$ & $\mathbf{K}$ & $\mathbf{C a}$ & $\mathrm{Mg}$ \\
\hline & \multirow[t]{2}{*}{$\mathrm{Mg} \mathrm{ha}^{-1}$} & \multirow{2}{*}{\multicolumn{4}{|c|}{$\begin{array}{l}-\mathrm{kg} \mathrm{ha}^{-1}- \\
\text { meadura }\end{array}$}} & \\
\hline & & & & & & \\
\hline Crotalária juncea & - & - & - & - & - & - \\
\hline Mucuna cinza & $0,25 b^{(1)}$ & $5,8 \mathrm{~b}$ & $0,3 \mathrm{~b}$ & $1,8 \mathrm{~b}$ & $2,5 \mathrm{~b}$ & $2,2 \mathrm{c}$ \\
\hline Feijão-de-porco & $0,46 \mathrm{a}$ & $14,1 \mathrm{a}$ & $0,4 \mathrm{a}$ & $4,4 \mathrm{a}$ & $10,1 \mathrm{a}$ & $6,5 \mathrm{a}$ \\
\hline Mucuna preta & $0,10 \mathrm{c}$ & $1,9 \mathrm{c}$ & $0,1 \mathrm{c}$ & $0,5 \mathrm{c}$ & $1,1 \mathrm{~b}$ & $0,7 \mathrm{~b}$ \\
\hline Lab-lab & $0,11 \mathrm{c}$ & $2,2 \mathrm{c}$ & $0,1 \mathrm{c}$ & $1,0 \mathrm{c}$ & $1,3 \mathrm{~b}$ & $1,0 \mathrm{~b}$ \\
\hline Crotalaria spectabilis & - & - & - & - & - & - \\
\hline Guandu anão & - & - & - & - & - & - \\
\hline \multirow[t]{2}{*}{ CV $(\%)$} & 23,97 & 18,38 & 35,03 & 25,04 & 29,99 & 28,33 \\
\hline & \multicolumn{5}{|c|}{90 dias após a semeadura } & \\
\hline Crotalária juncea & $0,41 \mathrm{~d}$ & $6,4 \mathrm{~d}$ & $0,36 \mathrm{~d}$ & $2,2 \mathrm{c}$ & $7,8 \mathrm{c}$ & $5,8 \mathrm{~b}$ \\
\hline Mucuna cinza & $1,53 \mathrm{a}$ & $33,9 \mathrm{a}$ & $1,65 \mathrm{a}$ & $8,2 \mathrm{~b}$ & $17,2 \mathrm{a}$ & $13,6 \mathrm{a}$ \\
\hline Feijão-de-porco & $0,66 \mathrm{c}$ & $17,5 \mathrm{c}$ & $0,32 \mathrm{~d}$ & $8,5 \mathrm{~b}$ & $9,8 \mathrm{~b}$ & $5,7 \mathrm{~b}$ \\
\hline Mucuna preta & $0,72 \mathrm{c}$ & $23,1 \mathrm{~b}$ & $0,81 \mathrm{c}$ & $4,5 \mathrm{c}$ & $8,4 \mathrm{c}$ & $6,7 \mathrm{~b}$ \\
\hline Lab-lab & $1,24 \mathrm{~b}$ & $24,6 \mathrm{~b}$ & $1,19 \mathrm{~b}$ & $15,4 \mathrm{a}$ & $19,7 \mathrm{a}$ & $15,4 \mathrm{a}$ \\
\hline Crotalaria spectabilis & $0,64 \mathrm{c}$ & $15,4 \mathrm{c}$ & $0,45 \mathrm{~d}$ & $7,2 \mathrm{~b}$ & $12,4 \mathrm{~b}$ & $5,0 \mathrm{~b}$ \\
\hline Guandu anão & $0,75 \mathrm{c}$ & $18,7 \mathrm{c}$ & $0,56 \mathrm{~d}$ & $2,4 \mathrm{c}$ & $6,0 \mathrm{c}$ & $3,3 \mathrm{~b}$ \\
\hline \multirow{2}{*}{$\mathrm{CV}(\%)$} & 13,21 & 16,18 & 22,99 & 23,40 & 15,90 & 21,29 \\
\hline & \multicolumn{5}{|c|}{120 dias após a semeadura } & - \\
\hline Mucuna cinza & $2,6 \mathrm{a}$ & $62,6 \mathrm{a}$ & $4,4 \mathrm{a}$ & $9,6 \mathrm{a}$ & $28,0 \mathrm{a}$ & $22,8 \mathrm{a}$ \\
\hline Feijão-de-porco & - & - & - & - & - & - \\
\hline Mucuna preta & $1,6 \mathrm{~b}$ & $44,7 \mathrm{~b}$ & $1,8 \mathrm{~b}$ & $3,3 \mathrm{~b}$ & $21,0 \mathrm{a}$ & $11,9 \mathrm{~b}$ \\
\hline Lab-lab & $1,4 \mathrm{~b}$ & $35,6 \mathrm{~b}$ & $1,7 \mathrm{~b}$ & $5,4 \mathrm{~b}$ & $21,8 \mathrm{a}$ & $13,3 \mathrm{~b}$ \\
\hline Crotalaria spectabilis & - & - & - & - & - & - \\
\hline Guandu anão & - & - & - & - & - & - \\
\hline $\mathrm{CV}(\%)$ & 21,63 & 23,14 & 15,56 & 24,73 & 16,77 & 19,08 \\
\hline
\end{tabular}

(1) Valores seguidos de letras iguais, dentro da coluna, não diferem entre si pelo teste de Scott-Knott $(p<0,05)$. 
que apresentaram aporte significativo de material senescente foram feijão-de-porco, mucuna-cinza, lablabe e mucuna-preta. As leguminosas arbustivas, guandu-anão, Crotalaria juncea e Crotalaria spectabilis, não contribuíram com aporte de material senescente nesse período. Todas as espécies contribuíram com material senescente aos 90 DAS. Contudo, aos 120 DAS, somente mucuna-cinza, mucuna-preta e lablabe continuaram a contribuir com a matéria seca senescente sobre o solo.

Pelos dados, observa-se que as espécies leguminosas mucuna-cinza, lablabe e mucuna-preta apresentaram maior potencial para deposição de material senescente sobre o solo e, com isso, potencial para o aporte de nutrientes, com destaque para a primeira. Isso denota o potencial para utilização dessa espécie como planta companheira durante o ciclo de culturas de interesse econômico, podendo contribuir significativamente para enriquecimento e aporte de nutrientes e aumento da matéria orgânica sobre o solo, favorecendo dessa forma a introdução dela em consórcio com culturas. Os resultados obtidos na taxa de cobertura do solo, em conjunto com a deposição de material senescente, demonstram que a mucuna-cinza apresenta características relevantes e de grande importância na proteção dos solos e cultivo em consórcio.

Nas avaliações realizadas no florescimento das plantas (Quadro 3), para os teores de N não houve diferenças significativas entre as espécies. No entanto, quando se observam os teores de N na parte aérea na presença dos frutos, notam-se diferenças entre as espécies. Isso ocorre porque para algumas delas, que foi o caso de mucuna-cinza, mucuna-preta e lablabe, os teores de $\mathrm{N}$ encontrados na parte aérea no momento do florescimento não se diferencia do teor de $\mathrm{N}$ na parte aérea na presença dos frutos, enquanto para as outras espécies esse teor diminui.

Quanto aos teores de P, nota-se que as espécies mucuna-cinza, lablabe e guandu-anão apresentaram os maiores valores no momento do florescimento.
Quando observado na frutificação, nota-se que a maioria das espécies reduziu o teor de P na parte aérea, exceto Crotalaria juncea e Crotalaria spectabilis, o que pode ser uma característica intrínseca do gênero. $\mathrm{O}$ guandu-anão, apesar da redução no teor de $\mathrm{P}$, ainda foi o que mostrou maiores valores na presença dos frutos, quando comparado com as outras espécies (Quadro 3). Isso ocorre pelo fato de que as raízes do guandu, além de diferenciadas e profundas, exsudam ácidos orgânicos, principalmente o cítrico, que agem na solubilização do $\mathrm{P}$ ligado ao $\mathrm{Ca}$, o que favorece a absorção deste (Ae et al., 1990).

Para o comportamento apresentado pelas leguminosas quanto ao $\mathrm{K}$ na fase de flor, merecem destaque o feijão-de-porco e a Crotalaria spectabilis. Quando observados os teores na fase de frutificação, observa-se que apenas a Crotalaria spectabilis se destaca. Todavia, nota-se queda nos teores de K entre os estádios vegetativos das plantas (Quadro 3). Com relação ao $\mathrm{Ca}$ e $\mathrm{Mg}$, os resultados foram similares, com a maioria das espécies apresentando maiores teores na fase de frutificação (Quadro 3).

Em relação aos teores de N, P e K, a tendência foi de diminuir na fase de frutificação. Para o N, as mucunas não se diferenciaram estatisticamente nas fases de florescimento e frutificação; esse fato pode ser justificado pelo comportamento durante o seu desenvolvimento, pois elas não floresceram de forma homogênea, o que pode ter influenciado no resultado. No geral, os teores de N, P e K são mais elevados no florescimento, com as quantidades diminuindo nas avaliações no período de frutificação. Para os teores de Ca, observa-se tendência de aumento deles na matéria seca das leguminosas. Com relação ao Mg, tem-se um comportamento diferente entre as espécies, ocorrendo aumento do teor desse nutriente na fase de frutificação, exceto para feijão-de-porco e Crotalaria spectabilis. O Ca, por ser elemento de baixa mobilidade no floema dos tecidos, apresenta tendência de acúmulo, aumentando a concentração com o tempo

Quadro 3. Teores de nitrogênio, fósforo, potássio, cálcio e magnésio na parte aérea das leguminosas, em diferentes fases do ciclo vegetativo

\begin{tabular}{|c|c|c|c|c|c|c|c|c|c|c|}
\hline \multirow{2}{*}{ Espécie } & \multicolumn{2}{|c|}{$\mathbf{N}$} & \multicolumn{2}{|c|}{$\mathbf{P}$} & \multicolumn{2}{|c|}{$\mathbf{K}$} & \multicolumn{2}{|c|}{$\mathbf{C a}$} & \multicolumn{2}{|c|}{ Mg } \\
\hline & Flor & Fruto & Flor & Fruto & Flor & Fruto & Flor & Fruto & Flor & Fruto \\
\hline & & & & & & $\mathrm{kg}^{-1}$ & & & & \\
\hline $\mathrm{Cj}$ & $37 \mathrm{Aa}^{(1)}$ & $30 \mathrm{Bb}$ & $2,0 \mathrm{Ab}$ & $1,8 \mathrm{Ab}$ & $11,9 \mathrm{Ac}$ & $8,4 \mathrm{Bc}$ & $8,9 \mathrm{Ad}$ & $10,1 \mathrm{Ab}$ & $8,1 \mathrm{Bc}$ & $10,8 \mathrm{Aa}$ \\
\hline $\mathrm{Mc}$ & $36 \mathrm{Aa}$ & $37 \mathrm{Aa}$ & $2,9 \mathrm{Aa}$ & $1,5 \mathrm{Bc}$ & $14,1 \mathrm{Ab}$ & $4,5 \mathrm{Bd}$ & $7,4 \mathrm{Be}$ & $9,7 \mathrm{Ab}$ & $7,7 \mathrm{Bd}$ & $10,5 \mathrm{Aa}$ \\
\hline $\mathrm{Fp}$ & $38 \mathrm{Aa}$ & $30 \mathrm{Bb}$ & $2,0 \mathrm{Ab}$ & $1,4 \mathrm{Bc}$ & $16,5 \mathrm{Aa}$ & $9,8 \mathrm{Bb}$ & $13,1 \mathrm{Aa}$ & $12,1 \mathrm{Aa}$ & $11,7 \mathrm{Aa}$ & $8,4 \mathrm{Bb}$ \\
\hline $\mathrm{Mp}$ & $38 \mathrm{Aa}$ & $40 \mathrm{Aa}$ & $2,3 \mathrm{Aa}$ & $1,5 \mathrm{Bc}$ & $10,8 \mathrm{Ad}$ & $9,9 \mathrm{Ab}$ & $7,8 \mathrm{Be}$ & $8,7 \mathrm{Ab}$ & $5,9 \mathrm{Be}$ & $10,5 \mathrm{Aa}$ \\
\hline $\mathrm{Ll}$ & $35 \mathrm{Aa}$ & $38 \mathrm{Aa}$ & $2,7 \mathrm{Aa}$ & $1,8 \mathrm{Bb}$ & $13,7 \mathrm{Ab}$ & $5,6 \mathrm{Bd}$ & $11,1 \mathrm{Ab}$ & $12,2 \mathrm{Aa}$ & $10,4 \mathrm{Ab}$ & $10,6 \mathrm{Aa}$ \\
\hline $\mathrm{Ce}$ & $37 \mathrm{Aa}$ & $28 \mathrm{Bb}$ & $1,6 \mathrm{Ab}$ & $1,4 \mathrm{Ab}$ & $15,4 \mathrm{Aa}$ & $12,5 \mathrm{Aa}$ & $10,2 \mathrm{Ac}$ & $11,3 \mathrm{Aa}$ & $7,2 \mathrm{Ad}$ & $6,0 \mathrm{Bc}$ \\
\hline $\mathrm{Ga}$ & $33 \mathrm{Aa}$ & $30 \mathrm{Bb}$ & $2,7 \mathrm{Aa}$ & $2,3 \mathrm{Ba}$ & $9,1 \mathrm{Ae}$ & $8,1 \mathrm{Bc}$ & $8,6 \mathrm{Bd}$ & $9,5 \mathrm{Ab}$ & $7,2 \mathrm{Bd}$ & $8,9 \mathrm{Ab}$ \\
\hline CV (\%) & 7,34 & 7,05 & 12,95 & 12,73 & 5,64 & 13,36 & 4,33 & 12,60 & 4,90 & 6,54 \\
\hline
\end{tabular}

(1) Valores seguidos de letras iguais, maiúsculas nas linhas e minúsculas nas colunas, não diferem entre si pelo teste de ScottKnott (p < 0,05). Esp.: Espécie; Cj: Crotalaria juncea; Mc: mucuna-cinza; Fp: feijão-de-porco; Mp: mucuna-preta; LL: lablabe; Ce: Crotalaria spectabilis; Ga: guandu-anão; Flor.: floração; Frut.: frutificação. 
(Marschner, 1995). Os resultados reforçam o fato de que a floração é o estádio vegetativo mais indicado para efetuar o manejo dos adubos verdes, quando se pretende disponibilizar quantidade significativa de $\mathrm{N}$, $\mathrm{P}$ e K aos agroecossistemas. Entretanto, com relação ao $\mathrm{Ca}$ e Mg, poderia ser mais vantajoso realizar o corte delas na fase de frutificação, quando se almeja maior aporte desses nutrientes dentro do sistema agrícola.

Com relação à produção de matéria seca, houve diferenças significativas entre as leguminosas; Crotalaria juncea destacou-se com a maior média, em relação às demais (Quadro 4). Os valores alcançados por essa espécie foram inferiores aos obtidos por Amabile et al. (2000) sob condições edafoclimáticas semelhantes, porém superiores aos encontrados por Oliveira \& Gosch (2007), também em região de Cerrado, e por outros autores em variadas regiões, como Castro et al. (2004), Cazetta et al. (2005) e Silva et al. (2006). A matéria seca acumulada é significativa e expressa o potencial da Crotalaria juncea para uso como cobertura em solos de Cerrado na região, pois esta pode proporcionar eficiente cobertura do solo pelos seus resíduos, permanecendo estes por maior tempo na superfície em relação às outras leguminosas, devido à alta relação $\mathrm{C} / \mathrm{N}(29)$ do seu material (Carvalho \& Amabile, 2006). Das espécies estudadas, o guanduanão apresentou a menor produção de matéria seca, que pode estar associada à baixa germinação das sementes e ao incidente ocorrido com o pastejo dos bovinos em algumas parcelas do experimento, que prejudicou o desenvolvimento da espécie e a formação do estande. Apesar desse ocorrido, os resultados foram superiores aos encontrados por Teixeira et al. (2005) e inferiores aos observados por Moreira et al. (2003) e Suzuki \& Alves (2006).

De acordo com Alvarenga et al. (2001), o acúmulo de $6 \mathrm{Mg} \mathrm{ha}^{-1}$ é uma quantidade de matéria seca que proporciona boa taxa de cobertura do solo, o que torna Crotalaria juncea, mucuna-cinza, feijão-de-porco e mucuna-preta leguminosas promissoras para adubação verde na região, pela capacidade de acumular matéria seca (Quadro 4).
No que se refere à quantidade de nutrientes acumulada na matéria seca das espécies, notaram-se diferenças significativas entre elas. Quanto ao N, notase que Crotalaria juncea foi a que acumulou as maiores quantidades, seguida por mucuna-cinza, feijão-de-porco e mucuna-preta, que não diferiram entre si, com Crotalaria spectabilis, lablabe e guanduanão apresentando os menores valores (Quadro 4).

No tocante aos conteúdos de $\mathrm{N}$ oriundos da fixação biológica de $\mathrm{N}_{2}$ (FBN), Castro et al. (2004) identificaram que, do total de $\mathrm{N}$ acumulado em Crotalaria juncea, a FBN contribuiu com $67 \%$; Moreira et al. (2003) observaram que, em média, 61 \% do N acumulado pelo guandu-anão provém da FBN; já mucunapreta, mucuna-cinza, Crotalaria spectabilis e lablabe teriam, em média, $70 \%$ do $\mathrm{N}$ total proveniente da FBN, segundo afirmativa de Giller (2001). Tomando como referência esses valores, pode-se estimar que os valores de $\mathrm{N}$ aportados via FBN pelas leguminosas, no presente trabalho, variaram de 53 a $344 \mathrm{~kg} \mathrm{ha}^{-1}$, representando quantidade suficiente para suprir as necessidades de $\mathrm{N}$ de uma gama de culturas.

$\mathrm{O}$ acúmulo de $\mathrm{P}$ foi maior para mucuna-cinza e Crotalaria juncea, que não diferiram estatisticamente entre si, e sim em relação às demais (Quadro 4). O acúmulo pela Crotalaria juncea está diretamente ligado à alta produção de matéria seca, enquanto para mucuna-cinza, segundo Sodré Filho et al. (2004), pode estar associada às características de elevada produção de matéria seca, raízes profundas e a possíveis colonizações radiculares por fungos micorrízicos arbusculares nativos, o que ocorre naturalmente (Miranda \& Miranda, 2001).

Quanto ao acúmulo de K, destacaram-se Crotalaria juncea, feijão-de-porco e mucuna-cinza (Quadro 4). A alta capacidade de acúmulo de $\mathrm{K}$ apresentada por essas leguminosas torna-as uma boa alternativa para incremento desse elemento em sistemas nos quais sejam cultivadas principalmente espécies exigentes nesse nutriente - como é o caso das espécies acumuladoras de amido em partes subterrâneas, como a mandioca, que é de suma importância na região.

Quadro 4. Acúmulo de matéria seca e macronutrientes em diferentes leguminosas na parte aérea, no período de florescimento

\begin{tabular}{|c|c|c|c|c|c|c|}
\hline Espécie & Matéria seca & $\mathbf{N}$ & $\mathbf{P}$ & $\mathbf{K}$ & $\mathbf{C a}$ & Mg \\
\hline & $\mathrm{Mg} \mathrm{ha}^{-1}$ & & & $\mathrm{~kg} \mathrm{ha}^{-1}$ & & \\
\hline Crotalária juncea & $13,90 \mathrm{a}^{(1)}$ & $514,00 \mathrm{a}$ & $27,22 \mathrm{a}$ & $165,51 \mathrm{a}$ & $123,55 \mathrm{a}$ & $111,83 \mathrm{a}$ \\
\hline Mucuna cinza & $9,80 \mathrm{~b}$ & $358,77 \mathrm{~b}$ & $29,01 \mathrm{a}$ & $138,53 \mathrm{a}$ & $73,03 \mathrm{~b}$ & $75,26 \mathrm{~b}$ \\
\hline Feijão-de-porco & $8,77 \mathrm{~b}$ & $335,60 \mathrm{~b}$ & $17,68 \mathrm{~b}$ & $146,39 \mathrm{a}$ & $114,68 \mathrm{a}$ & $102,42 \mathrm{a}$ \\
\hline Mucuna preta & $7,50 \mathrm{c}$ & $286,10 \mathrm{~b}$ & $17,34 \mathrm{~b}$ & $80,86 \mathrm{~b}$ & $58,52 \mathrm{~b}$ & $44,10 \mathrm{c}$ \\
\hline Lab-lab & $5,65 \mathrm{c}$ & $198,32 \mathrm{c}$ & $15,35 \mathrm{~b}$ & $76,95 \mathrm{~b}$ & $62,69 \mathrm{~b}$ & $58,37 \mathrm{c}$ \\
\hline Crotalaria spectabilis & $5,45 \mathrm{c}$ & $201,00 \mathrm{c}$ & $8,71 \mathrm{c}$ & $84,17 \mathrm{~b}$ & $55,89 \mathrm{~b}$ & $39,27 \mathrm{c}$ \\
\hline Guandu anão & $2,62 \mathrm{~d}$ & $87,00 \mathrm{~d}$ & $7,12 \mathrm{c}$ & $23,70 \mathrm{c}$ & $22,54 \mathrm{c}$ & $18,78 \mathrm{~d}$ \\
\hline $\mathrm{CV}(\%)$ & 18,47 & 21,96 & 24,44 & 23,51 & 20,32 & 22,46 \\
\hline
\end{tabular}

(1) Valores seguidos de letras iguais, dentro da coluna, não diferem entre si pelo teste de Scott-Knott $(p<0,05)$. 
As espécies Crotalaria juncea e feijão-de-porco não diferiram entre si com relação ao acúmulo de Ca e $\mathrm{Mg}$, porém diferiram das outras leguminosas, apresentando os maiores valores (Quadro 4). Os valores observados em todas as espécies foram superiores aos verificados por Amabile et al. (2000), também trabalhando em solos de Cerrado. Essa alta capacidade de aporte de $\mathrm{Mg}$ e Ca pelas espécies pode estar relacionada aos benefícios da calagem sobre o crescimento e absorção dos nutrientes pelas leguminosas, o que está associado principalmente ao aumento na disponibilidade de $\mathrm{Ca}$ e $\mathrm{Mg}$ às plantas (Carvalho et al., 1988).

Esses resultados reforçam o potencial para o uso de leguminosas na adubação verde, sobretudo das espécies estudadas, como alternativa para economia substancial de fertilizantes, particularmente importantes para os agricultores familiares da região e de outras regiões, que normalmente são descapitalizados e muitas vezes cultivam em solos depauperados, devido aos anos de exploração ou à escassez de reservas de nutrientes. Outro aspecto de grande dimensão é a contribuição ambiental, como a proteção dos solos, reduzindo perdas por processos como a lixiviação, o carreamento de partículas por erosão e outros, que promovem perdas na qualidade dos solos, sobretudo em ambientes tropicais.

\section{CONCLUSÕES}

1. O ciclo precoce de Crotalaria juncea, Crotalaria spectabilis e feijão-de-porco favorece a inserção destas nos sistemas de cultivo com rotação e sucessão de culturas para o solo argiloso do Cerrado do Alto Vale do Jequitinhonha.

2. O manejo das leguminosas no período do florescimento é o mais recomendado, para o maior aporte de $\mathrm{N}, \mathrm{Pe} \mathrm{K}$ aos agroecossistemas.

3. Entre as leguminosas, as maiores produções de matéria seca foram de Crotalaria juncea, mucunacinza, feijão-de-porco e mucuna-preta, sendo estas indicadas para adubação verde nas condições de Cerrado estudadas.

4. As leguminosas avaliadas apresentam elevado potencial para o incremento na oferta de $\mathrm{N}$ aos sistemas de produção.

\section{AGRADECIMENTOS}

À CEART, pelo apoio oferecido na realização deste trabalho; à FAPEMIG, CNPq, MDA/SAF e MDS/ SESAN, pelo auxílio financeiro; e à UFVJM, pelo suporte à pesquisa.

\section{LITERATURA CITADA}

AE, N.; ARIHARA, K.; OKADA, K.; YOSIHARA, T. \& JOHANSEN, C. Phosphorus uptake by pigeonpea and its role in cropping systems of Indian Subcontinent. Science, 248:477-480, 1990.

ALVARENGA, R.C.; CABEZAS, W.A.L.; CRUZ, J.C. \& SANTANA, D.P. Plantas de cobertura de solo para sistema plantio direto. Inf. Agropec., 22:25-36, 2001.

AMABILE, R.F.; CARVAlho, A.M.; DUARTE, J.B. \& FANCELLI, A.L. Efeito de épocas de semeadura na fisiologia e produção de matéria seca de leguminosas nos Cerrados da região do Mato Grosso de Goiás. Sci. Agric., 53:296-303, 1996.

AMABILE, R.F.; FANCELLI, A.L. \& CARVALHO, A.M. Comportamento de espécies de adubos verdes em diferentes épocas de semeadura e espaçamentos na região dos Cerrados. Pesq. Agropec. Bras., 35:47-54, 2000.

ARF, O.; BUZETTI, S.; ALVES, M.C.; SÁ, M.E.; RODRIGUES, R.A.F. \& HERNANDEZ, F.B.T. Efeito da época de semeadura da mucuna-preta (Stizolobium aterrimum) e lablabe (Dolichos lablab) intercalados na cultura do milho (Zea mays). Ci. Agrotec., 24:898:904, 2000.

BATAGLIA, O.C.; FURLANI, A.M.C.; TEIXEIRA, J.P.F.; FURLANI, P.R. \& GALLO, J.R. Método de análise química de plantas. B. Tecn. Inst. Agron., 78:1-48, 1983.

BREMNER, J.M. \& MULVANEY, C.S. Nitrogen total, In: PAGE, A.L.; MILLER, R.A. \& KEENEY, D.R., eds. Methods of soil analysis. 2.ed. Madison, American Society of Agronomy, 1982. p. 595-624. (Agronomy, 9)

CALEGARI, A.; ALCANTRA, P.B.; MIYASAKA, S. \& AMADO, T.J.C. Características das principais espécies de adubos verdes. In: COSTA, M.B.B., coord. Adubação verde no Sul do Brasil. Rio de Janeiro, AS-PTA, 1993. p.206-319.

CALEGARI, A. Leguminosas para adubação verde no Paraná. Curitiba, IAPAR, 1995.

CARNEIRO, M.A.C.; CORDEIRO, M.A.S.; ASSIS, P.C.R.; MORAES, E.S.; PEREIRA, H.S.; PAULINO, H.B. \& SOUZA, E.D. Produção de matéria seca de diferentes espécies de cobertura e suas alterações na atividade microbiana de solo de cerrado. Bragantia, 67:455-462, 2008.

CARVALHO, A.M. \& AMABILE, R.F. Cerrado: Adubação verde. Brasília, Embrapa Cerrados, 2006. 369p.

CARVALHO, A.M. Uso de plantas condicionadoras com incorporação e sem incorporação no solo: Composição química e decomposição de resíduos vegetais; disponibilidade de fósforo e emissão de gases. Brasília, Universidade de Brasília, 2005. 199p. (Tese de Doutorado)

CARVALHO, A.M.; BURLE, M.L.; PEREIRA, J. \& SILVA, M.A. Manejo de adubos verdes no Cerrado. Embrapa Cerrados, 1999. 28p. (Circular Técnica, 4)

CARVALHO, M.M.; SARAIVA, O.F.; OLIVEIRA, F.T.T. \& MARTINS, C.E. Respostas de leguminosas forrageiras tropicais à calagem e ao fósforo, em casa de vegetação. R. Bras. Ci. Solo, 12:153-159, 1988. 
CASTRO, C.M.; ALVES, B.J.R.; ALMEIDA, D.L. \& RIBEIRO, R.L.D. Adubação verde como fonte de $\mathrm{N}$ para a cultura da berinjela em sistema orgânico. Pesq. Agropec. Bras., 39:779-785, 2004.

CAZETTA, D.A.; FORNASIERI FILHO, D. \& GIROTTO, F. Composição, produção de matéria seca e cobertura do solo em cultivo exclusivo e consorciado de milheto e crotalária. Acta Sci. Agron., 27:575-580, 2005.

CHAVES, J.C.D. \& CALEGARI, A. Adubação verde e rotação de culturas. Inf. Agropec., 22:53-60, 2001.

DAROLT, M.R. Princípios para manutenção e implantação do sistema. In: DAROLT, M.R. Plantio direto: Pequena propriedade sustentável. Curitiba, IAPAR, 1998. (Circular. 101)

EMPRESA BRASILEIRA DE PESQUISA AGROPECUÁRIA EMBRAPA. Centro Nacional de Pesquisa de Solos. Sistema brasileiro de classificação de solos. Rio de Janeiro, 2006. 306p.

EMPRESA BRASILEIRA DE PESQUISA AGROPECUÁRIA EMBRAPA. Centro Nacional de Pesquisa de Solos. Manual de métodos de análise de solo. Rio de Janeiro, 1997.

FÁVERO, C.; JUCKSCH, I.; ALVARENGA, R.C. \& COSTA, L.M. Modificações na população de plantas espontâneas na presença de adubos verdes. Pesq. Agropec. Bras., 36:1355-1362, 2001.

FERREIRA, D.F. SISVAR: Um programa para análises e ensino de estatística. R. Symposium, 6:36-41, 2008.

GILLER, K.E. Nitrogen fixation in tropical cropping systems. 2.ed. Wallingford, CBA International, 2001. 448p.

LEITE, L.F.C.; FREITAS, R.C.A.; SAGRILO, E. \& GALVÃO, S.R.S. Decomposição e liberação de nutrientes de resíduos vegetais depositados sobre Latossolo Amarelo no Cerrado Maranhense. R. Ci. Agron., 41:29-35, 2010.

MARSCHNER, H. Mineral nutrition of higher plants. London, Academic Press, 1995. 882p.

MIRANDA, J.C.C. \& MIRANDA, L.N. Manejo de micorriza arbuscular por meio da rotação de culturas nos sistemas agrícolas do cerrado. Brasília, Embrapa Cerrados, 2001. 3p. (Comunicado Técnico, 42)

MOREIRA, V.F.; PEREIRA, A.J.; GUERRA, J.G.M.; GUEDES, R.E. \& COSTA, J.R. Produção de biomassa de guandu em função de diferentes densidades e espaçamentos entre sulcos de plantio. Seropédica, Embrapa Agrobiologia, 2003. 5p (Comunicado Técnico, 57)

OLIVEIRA, F.L. \& GOSCH, M.S. Potencial de leguminosas herbáceas de hábito ereto para adubação verde no cerrado do Tocantis. R. Ci. Agroamb., 2:17-24, 2007.
PERIN, A.; TEIXEIRA, M.G. \& GUERRA, J.G.M. Desempenho de algumas leguminosas com potencial para utilização como cobertura permanente de solo. Agronomia, 34:3843, 2000.

SANTOS, V.S. \& CAMPELO JÚNIOR, J.H. Influência dos elementos meteorológicos na produção de adubos verdes, em diferentes épocas de semeadura. R. Bras. Eng. Agríc. Amb., 7:91-98, 2003.

SILVA, E.C.; MURAOKA, T.; BUZETTI, S.; VELOSO, M.E.C. \& TRIVELIN, P.C.O. Aproveitamento do nitrogênio da crotalária e do milheto pelo milho sob plantio direto em Latossolo Vermelho de Cerrado. Ci. Rural, 36:739-746, 2006.

SILVA, J.E.; LEMAINSKI, J. \& RESK, D.V.S. Perdas de matéria orgânica e suas relações com a capacidade de troca catiônica em solos da região de Cerrados do oeste baiano. R. Bras. Ci. Solo, 18:541-547, 1994.

SILVA, P.C.G.; FOLONI, J.S.S.; FABRIS, L.B. \& TIRITAN, C.S. Fitomassa e relação $\mathrm{C} / \mathrm{N}$ em consórcios de sorgo e milho com espécies de cobertura. Pesq. Agropec. Bras., 44:1504-1512, 2009.

SILVEIRA, P.M.; BRAZ, A.J.B.P.; KLIEMANN, H.J. \& ZIMMERMANN, F.J.P. Acumulação de nutrientes no limbo foliar de guandu e estilosantes. Pesq. Agropec. Trop., 35:133-138, 2005

SODRÉ FILHO, J.; CARDOSO, A.N.; CARMONA, R. \& CARVALHO, A.M. Fitomassa e cobertura do solo de culturas em sucessão ao milho na região do Cerrado. Pesq. Agropec. Bras., 39:327-334, 2004.

SUZUKI, L.E.A.S. \& ALVES, M.C. Fitomassa de plantas de cobertura em diferentes sucessões de culturas e sistemas de cultivo. Bragantia, 65:121-127, 2006.

TANAKA, R.T. Adubação verde. Inf. Agropec., 7:62-67, 1981.

TEIXEIRA, C.M.; CARVALHO, G.J.; FURTINI NETO, A.E.; ANDRADE, M.J.B. \& MARQUES, E.L.S. Produção de biomassa e teor de macronutrientes do milheto, feijãode-porco e guandu-anão em cultivo solteiro e consorciado. Ci. Agrotec., 29:93-99, 2005.

TORRES, J.L.R.; PEREIRA, M.G.; ANDRIOLI, I.; POLIDORO, J.C. \& FABIAN, A.J. Decomposição e liberação de nitrogênio de resíduos culturais de plantas de cobertura em um solo de cerrado. R. Bras. Ci. Solo, 29:609-618, 2005 .

WALLIS, E.S.; SAXENA, K.B. \& BYTH, D.E. Flowering responses of thirty-seven early maturing lines of pigeonpea. In: INTERNATIONAL WORKSHOP ON PIGEONPEAS, 1981, Patancheru. Proceedings... Patancheru, ICRISAT, 1981. v.2. p.143-150. 
